\title{
NEWEL APPROACHES FOR TARGETED SPRAYING IN ORCHARDS
}

\author{
STAJNKO, D.; Vindis, P. \& LAKOTA, M.
}

Abstract: Modern fruit orchards are treated with pesticides 15 to 25 times per season. The high frequency of pesticide application represents one of the most serious pollution threats to the neighboring environment among various agricultural production systems. More accurate targeted spraying is possible by detecting of tree canopy with different sensors and appropriate electronics. Ultra sound signal used in modern spraying equipment is processed by a personal computer and fed in real-time to spraying nozzles which open and close in relation to the canopy structure. The evaluation in field experiments by detecting deposits on leaves and water sensitive papers (WSP) demonstrates decreasing of the amount of delivered spray. Thereby significant reduction of costs and environmental pollution by plant protection products is possible.

Key words: air-assisted sprayer, ultra sound, algorithm, spray distribution, orchard
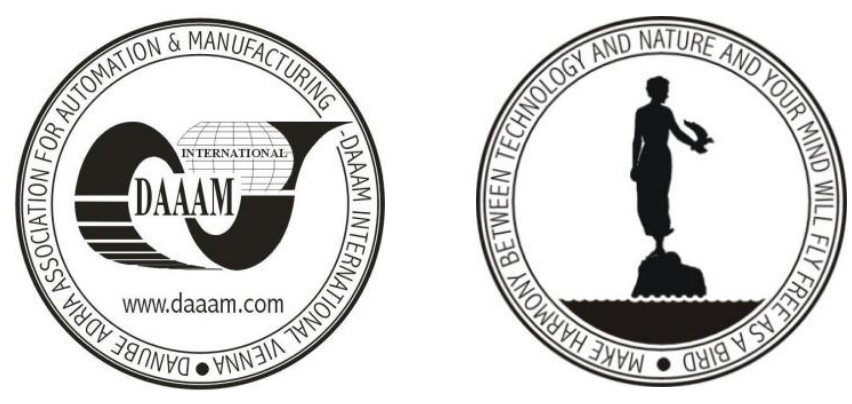

Authors' data: Assoc. Prof. Stajnko, D[enis], Assist. Prof. Vindis, P[eter], Assoc. Prof. Lakota, M[iran], University of Maribor, Faculty of Agriculture and Life Sciences, Pivola 10, SI-2311, Hoče, Slovenia, denis.stajnko@uni-mb.si, peter.vindis@uni-mb.si,miran.lakota@uni-mb.si

This Publication has to be referred as: Stajnko, D[enis]; Vindis, P[eter] \& Lakota, M[iran] (2013) Newel Approaches for Targeted Spraying in Orchards , Chapter 30 in DAAAM International Scientific Book 2013, pp. 561-570, B. Katalinic \& Z. Tekic (Eds.), Published by DAAAM International, ISBN 978-3-901509-94-0, ISSN 17269687, Vienna, Austria

DOI: 10.2507/daaam.scibook.2013.30 


\section{Introduction}

Apple fruit orchards are sprayed mainly with a single axial fan 'mistblower' sprayers, which can be fitted with ducted conveyors in the form of towers, a cannon or a series of flexible pneumatic tubes to direct the spray-laden air. These sprayers are simple, robust, reliable and of comparatively low cost in terms of purchase and operation. The objective of spraying is to deliver an effective, uniform dose of product to a target area in a safe and timely manner. Unfortunately, the large radial spray plume generated by axial fan orchard sprayers is prone to drift, thus 80 per cent of the product can be lost as vapor and droplet, run-off and any off-target deposition. Drift not only costs time and money but may reduce the effectiveness of the application and increase the risk of environmental contamination (Cross et al, 2001; Sutton \& Unrath, 1988). Possibilities of adapting the characteristics of air stream generated by axial fan sprayer to different tree canopies are quite limited.

Another reason for causing drift by conventional spraying in an orchard represents switching on at the beginning of a row and switching off at the end of the row. When the sprayer is switched on the applied amount of Plant Protection Products (PPP) is constant, regardless of the volume of the tree foliage.

A number of systems for adjusting the applied dose of plant protection products according to orchard structure have been developed in the past decades. Widely accepted is the Tree Row Volume (TRV) dosing system initiated by Solanelles et al., 2006. In this system, the dose applied to an orchard is varied by varying the spray volume at constant pesticide concentration in proportion to the TRV. The TRV $\left(\mathrm{m}^{3}\right.$ $\left.\mathrm{ha}^{-1}\right)$ is the volume of the tree canopy per unit of ground area $(=10000 \mathrm{x}$ crown height $\mathrm{x}$ crop width / row spacing). The TRV spray volume adjustment system has been adapted and tested for low volume spraying in several European countries (Walklate et al., 2002, Rovira-Más et al., 2005, Pergher \& Petris, 2008). In contrast to TRV model, McConnell, et al., 1983 and Gil, et al., 2007 proposed the use of leaf area measurements to improve the correlation between deposits given by different types of spraying equipment and types of hedgerow vineyards. However, the different shapes and sizes of tree canopies, even among the same variety in the orchard, require continual calculation of TRV and adjustment of the applied dose of pesticide to optimize the spray application efficiency (Moltó, et al., 2001).

For those reasons, in the last 10 years measurement of crop structure has been simplified by the development of a range of non-invasive optical and ultrasonic sampling techniques. This sprayer adjusts the amount of pesticides used, depending on the foliage volume of the trees. According to Zande et al. (2010) this principal is called Canopy Density Spraying (CDS). In particular, the development of a compact, tractor-mounted light and range detection system (LIDAR, Fig.1) has made it possible to take quick and detailed readings of crop structure (Llorens, et al., 2010). These are suitable for computational processing to calculate a wide range of summary parameters based on a probabilistic interpretation of light transmission and crop interception characteristics (Tumbo, et al., 2002). Such a system employs a pulse time-of-flight ranging method, with separate apertures (side-by-side) for an infrared laser diode transmitter and a matched diode light receiver. 

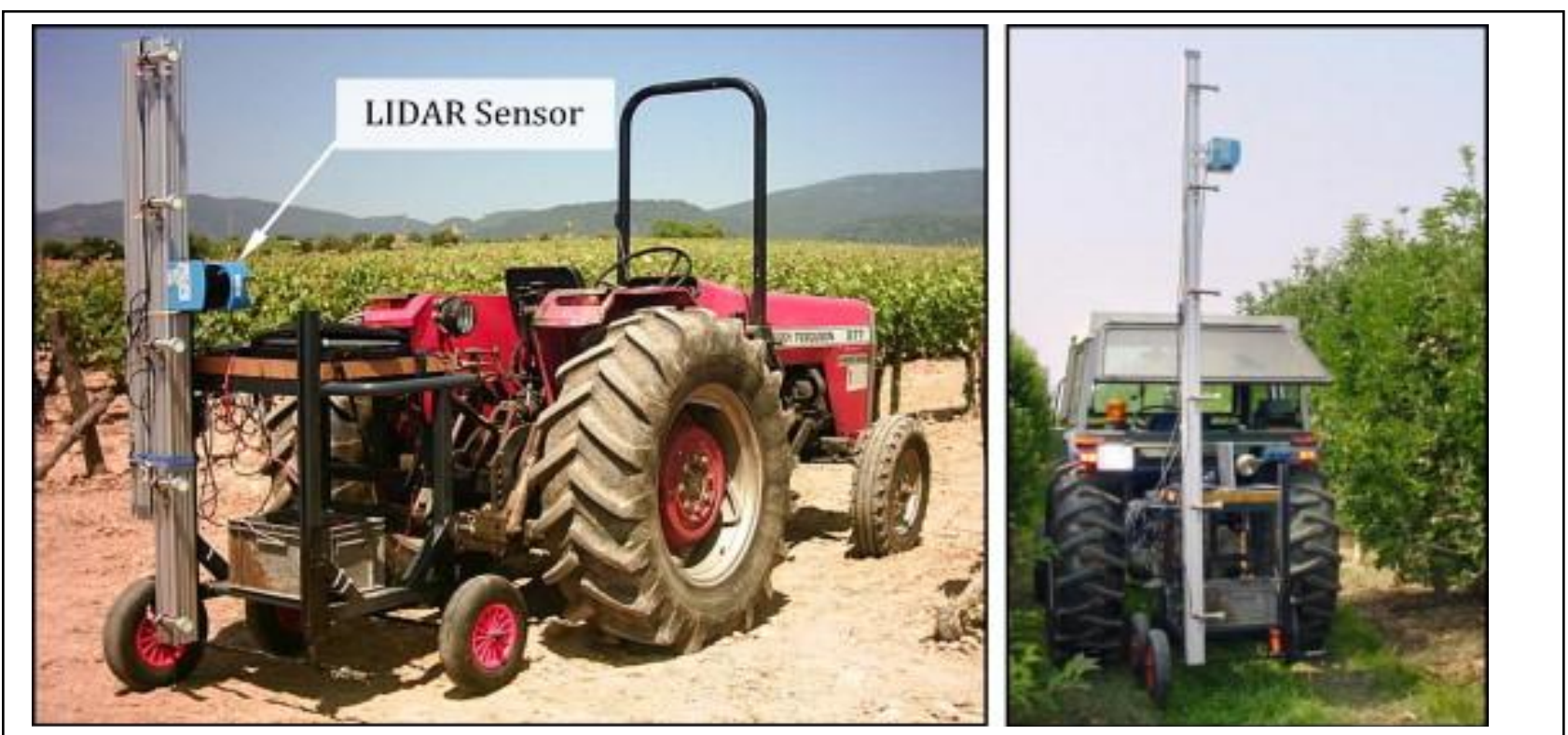

Fig. 1. LIDAR 3D dynamic measurement system (Rosell \& Sanz, 2012)

Contrary to the expensive radar system, Escolà et al. (2011) suggested the use of ultrasonic sensors and proportional electro-valves with the corresponding software and automation, which allowed real time modification of the sprayed flow rate adapted to the crop structure of the vineyard. In response to changes in the shape and size of the vines during the growing season, this system reduced the spray volume and the use of pesticides by up to $57 \%$, while maintaining coverage and penetration rates similar to those from conventional spraying methods.

However, since the ultrasonic sensors were originally designed to measure distances in industrial environments, where objects are rigid, and the surface of rebound is perpendicular to the direction of the ultrasonic wave, their utility in orchard measuring might be negligible (Koch \& Weisser, 2000, Walklate, et al., 2002). Some of the deficiencies of standard sensors can be overcome by modern sophisticated ultrasound signal processing algorithms.

In the following chapters we will present the development and testing of an automated orchard sprayer consisting of an axial fan with nozzles controlled by an ultrasound processing system. Results of experiments in the apple orchard and comparisons of spray coverage characteristics as well as the savings of spray between two working modes (with and without automated guidance) are presented in the following sections.

\section{Technical Background of Sensor Based Spraying}

For applying CDS, the characteristics of the canopy need to be measured which can be done with different sensors. Rosell \& Sanz (2012) studied seven different sensors for their usability under field conditions. From the different sensors, the stereo vision and the LIDAR are the most promising to get a 3D-map of canopies. However, the ultrasonic sensor remains a good option for certain applications, because their price and maintain. The ultrasonic sensor sends an ultrasound signal which reflects on any object in front of it. By determining the time requires for the 
sound to return to the sensor, the distance is calculated. An ultrasound sensor has a beam angle of $10-30^{\circ}$. The ultrasonic sensor performs well under general outdoor conditions; however the placing of the sensors on a sprayer needs to be done carefully. When a spray cloud moves in the reach of an ultrasonic sensor, it will trigger the sensor as well. Also, when multiple sensors are used there is a risk of interference (Jeon et al., 2011). Nowadays professional equipment for turning off/on nozzles at the end of the rows is commercially available by many sprayer producers. However the system is not able yet to adjust the sprayer prune inside the row according to the tree density or missing growing places. For this reason custom made sensors, built around microcontroller and connected to an ultrasonic transceiver is being tested recently. The use of microcontroller is required because a FPGA circuit enables to build a selective amplifier with a $40 \mathrm{~dB}$ gain and the frequency range of 40 $\mathrm{kHz}$, needed to amplify the received echoes. Usually for precise tree crown detection and density measurements more sensors is connected to the embedded computer, which provides cumulative responses over a predetermined range of half of row width $(\sim 1.50 \mathrm{~m})$ and a maximum response interval of $0.15 \mathrm{~m}$ inside a $1.50 \mathrm{~m}$ range. The applied microcontroller has to contain also a highly configurable analog section, which enables to build an intelligent sensor with serial output using only one chip the PSoC. The analog section of the PSoC is used for amplifying and band-pass filtering of the reflected ultrasonic signal. An envelope of the reflected signal is usually sampled with an 8-bit AD converter. Realizing both the analog and digital part of the sensor with one chip requires a higher clock frequency, which makes more noise in the analog section. Jejčič et al. (2011) applied a $6 \mathrm{MHz}$ clock, which enabled us to build a $40 \mathrm{~dB}$ gain amplifier for the reflected signal at sampling frequency of $15625 \mathrm{~Hz}$. This ultrasound system for targeted spraying in orchards was equipped with 3 nozzles on each side. For each nozzle there was an ultrasound sensor mounted to the sprayer. The nozzles were opened if there was a target in the range between 50 and $110 \mathrm{~cm}$. With this system a saving $20.2 \%$ was realized, without a significant reduction of spray coverage. The drawback of this system is that it only detects if there is a target, and not the volume or density of the tree canopy.

Instead of ultrasound, a laser scanner determines the distance to an object by emitting a light pulse. By obtaining the time it takes for the light pulse to return to the source, the distance can be calculated. The light pulse will be reflected by the first object it will run into (Zande et al., 2010). The beam angle used by a laser scanner is much smaller than the beam angle of an ultrasonic sensor. In general, the beam angle used by a laser scanner is less than $1^{\circ}$. The laser scanner rotates and takes multiple measurements, resulting in a fan-shaped scan of the environment. The information from the laser scanner needs to be processed in order to get the characteristics of the canopy. Different models that can be used to process these data into pesticide output are compared by Walklate et al. (2002). The models that were investigated used all a different way for calculating the pesticide output. Of these models the tree density model performed the best for a LIDAR guided sprayer.

There are different methods for calculating the surface of the canopy. Palacin et al. (2007) did analyze five different methods to calculate this. The canopy surface is used to calculate the volume of the tree, with the help of Cartesian projection. The 
volume of the tree is calculated with the measuring points of the laser and the assumed centre of the tree, by using a horizontally placed box. The centre of the tree, the measured points of the edge of the canopy and the tree spacing are the boundaries of the box. Together with the moved distance during the measurements the volume of the box is calculated. The simple Cartesian projection has the best correlation between tree volume and foliage surface.

The previous described CDS sprayers change the spray deposit by controlling the nozzles, whereas Pai et al. (2009) developed a system where the air flow of the sprayer is adjusted to the foliage density. The airflow rate of the sprayer is changed according to the foliage density in order to avoid spray losses. Therefore, the air flow is decreased when a lower density of the foliage is observed. A laser scanner and an algorithm are used to calculate the density of the foliage. Tests showed that a lower air speed results in a lower air penetration of the canopy. Changing the air flow has a bigger influence on high volume droplets than on low volume droplets. The test results indicate that changing the air flow rate may reduce spray deposit on the soil surface underneath and between trees in an orchard.

With a 2D LIDAR sensor 3D tree reconstruction can be done by using two measurements from opposing sides. This is much cheaper than using a 3D LIDAR. However, taking two measurements from opposing sides isn't suitable for in-line data-processing on a sprayer. Nevertheless, a single 2D LIDAR scan of a tree can be performed in-line. This system has a relative good accuracy in estimating the tree geometrics such as the surface area and volume of the canopy, despite its sensitivity to errors in the determination of the distance between the LIDAR-sensor and the centre of the tree (Sanz-Cortiella et al., 2011).

\section{Pesticide Dose in Orchards}

The standard method of pesticide dose expression in agriculture is expressed as a constant unit per ground area. The standard method of pesticide dose expression is well suited when a boom sprayer is used, but isn't suitable in cases of orchard spraying. The boom sprayer is most often used for pesticide application from above the crop, where a uniform horizontal distribution is preferred. When an orchard is sprayed, the horizontal distribution is not uniform at all (Walklate et al., 2002). Three methods for pesticide dose expression which are better suited for orchard spraying are the fruit wall area method (FWA), the TRV and the TAD method. The FWA method uses the fruit wall area to express the amount of pesticides used. A constant pesticide product per unit tree row volume per unit tree row length is used for the TRV method. And for the TAD the dose is expressed per unit area of the target (Walklate et al., 2002). From tests performed by Walklate et al. (2002), the tree area density model for dose expression looks the most promising for a constant average spray deposition rate at the leaves of a tree. In addition, Walklate et al. (2006) confirms that the TAD is the best model for adjusting the spray application rate.

Besides, this model can be improved by also taking the height of the tree row into account. Furthermore, the constant dose per hectare does not take the different row width into account. When orchard with different row widths are treated the spray 
Stajnko, D.; Vindis, P. \& Lakota, M.: Newel Approaches for Targeted Spraying in ...

volume per hectare needs to be adjusted with respect to the row width, to achieve a constant spray volume per FWA (Koch, 2007).

\section{Hardware and Software Design}

The application of sensing system during spraying is possible only with a programmable ultrasonic transceiver and an embedded computer. Experimental work under laboratory and orchard conditions needs to be conducted prior commercial application of each system. Sensing tests and the algorithm concepts are mainly set up in the laboratory, and then verified under the orchard condition.

First, the parameters of the programmable ultrasonic sensing system, such as reflected ultrasonic values, are derived in the orchard. Then, by using these parameters as thresholds, a spraying experiment is performed. For the spraying experiment, a modified axial sprayer is used, while operation is evaluated by deposit measurement by a composite leaf sample method. In the following paragraphs, design, algorithms of operation and experimental procedure will be discussed in detail.

Fig. 2 introduces the concept of interconnection between different parts of the system. On the left side of Fig. 2, there are three ultrasonic sensors that are triggered (orange line) by the LPC1343 microcontroller. The sensors then transmit readings by using a RS-232 connection (blue lines), coupled by the AND gate to a MAX232 lever shifter, which is connected to the UART (green line) of the microcontroller. The MAX232 is also connected to an Xbee Pro device that transmits or receives the data from the workstation wirelessly.

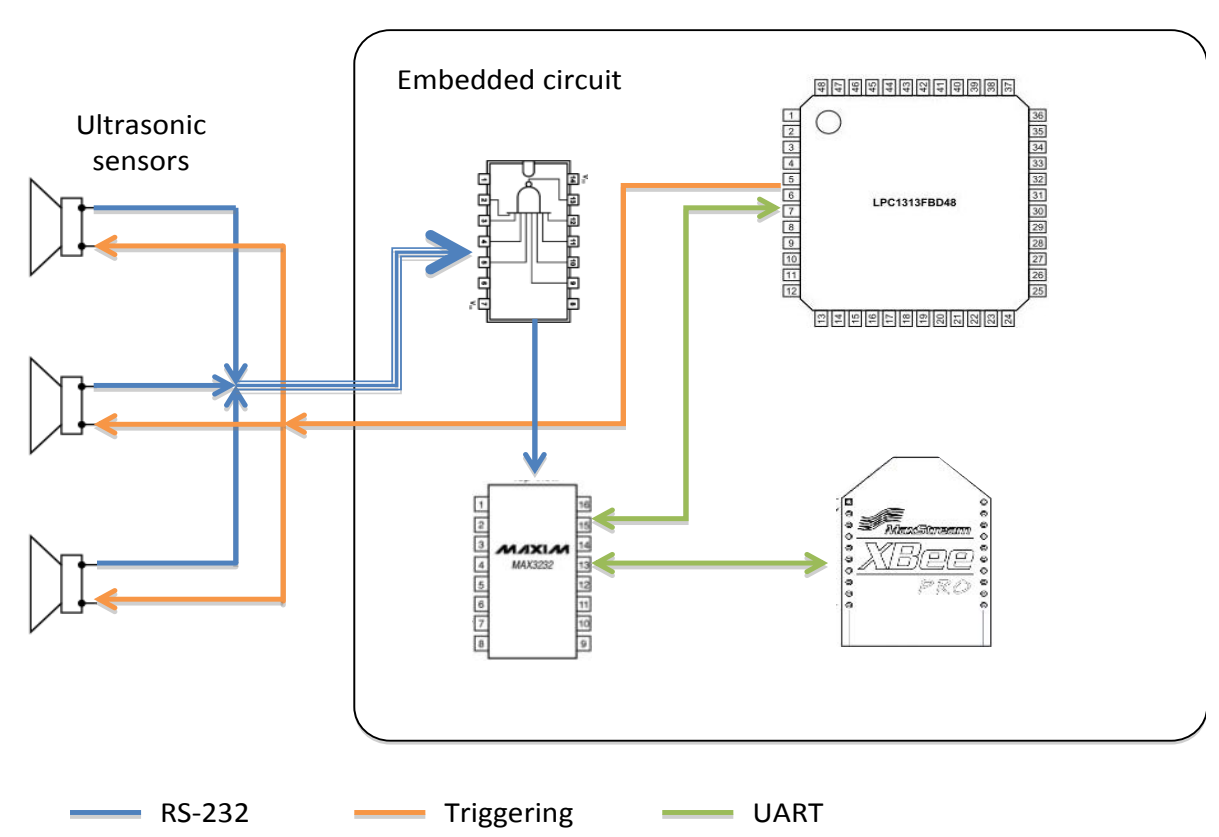

Fig. 2. Major components of the embedded circuit with the ultrasonic sensors (Jejčič et al. 2012). 


\subsection{Transceiver}

A sample of the ultrasonic transceivers (PROWAVE 400EP250) that is waterproof and generates a high sound pressure level is represented in the Fig 3. It may be used to transmit ultrasonic bursts and receive echoes that bounce off nearby objects. The transceiver has to be connected to the microcontroller, which triggered and analyzed the ultrasonic signals. Each transceiver is equipped with a horn, manufactured from black plastic. Ultrasonic transceivers are inserted into the horn with the angle of $25^{\circ}$, so that they are attached from behind with a permanently elastic silicone kit. The enclosure, to which the horn is attached is manufactured from aluminum, and housed sensor electronics in a watertight compartment. The sensor enclosure is mounted on the sprayer using two nuts.

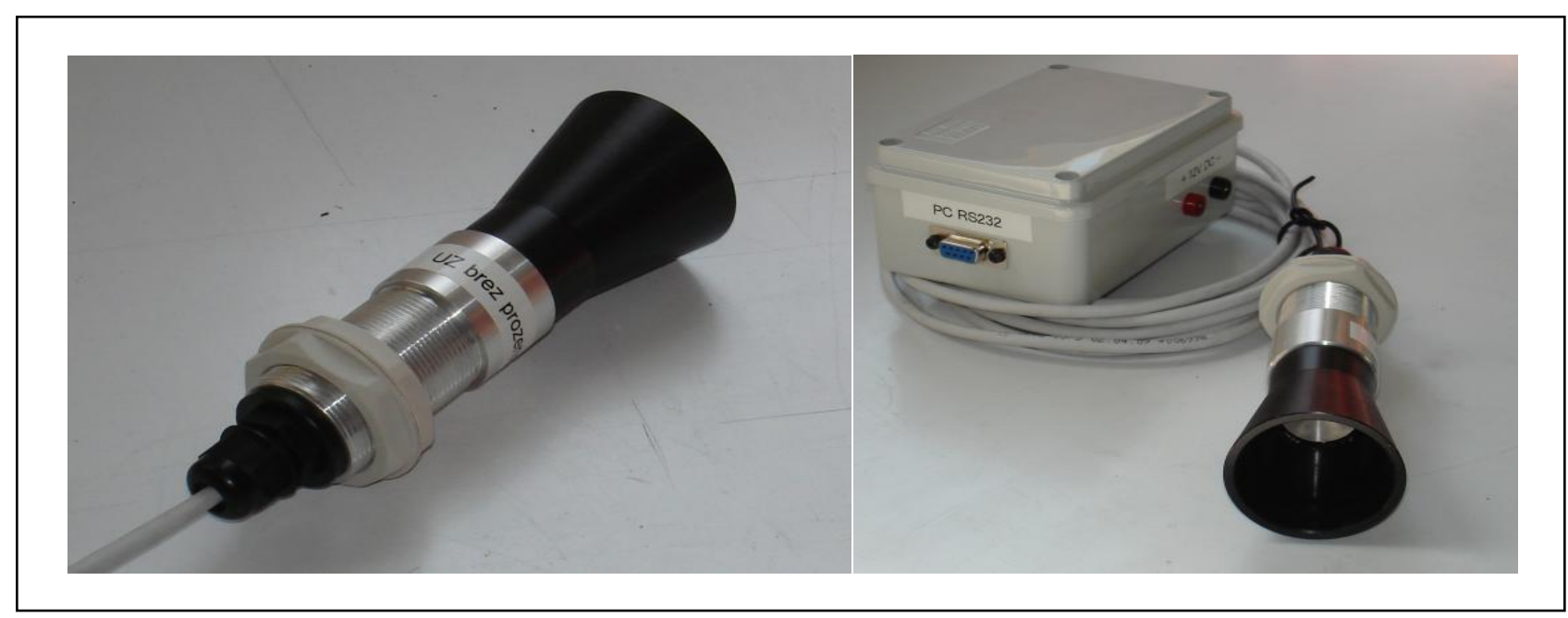

Fig. 3. Ultrasonic sensor with ultrasonic transceiver built in the horn. The enclosure includes sensor electronics (Jejčič et al. 2011).

\subsection{Sensing Algorithm}

Fig. 4 represents how the system works and the role of the LPC1343 microcontroller. After initialization and triggering of sensors, the system first reads the data and verifies the cumulative response; if it is high enough, the microcontroller proceeds with further analysis; otherwise, it continues with the next measurement. In the second stage we verify the interval maximum; if the interval maximum is too high and includes majority of the response, it measures the support column. In a case where the cumulative value is low and the maximum value is below the threshold, the microcontroller opens the nozzle at the height of the current sensor. The use of intelligent ultrasound based system in spraying usually requires time spanning from 0 to $25 \mathrm{~ms}$ per one loop of flowchart, which means that the canopy would be detected at definite distances i.e. from $2.2 \mathrm{~m}$ to $3.6 \mathrm{~m}$. In reality, the range is set from $0.45 \mathrm{~m}$ to $1.6 \mathrm{~m}$, which produced 9 samples and each corresponding to the thickness of the canopy at a given depth. 


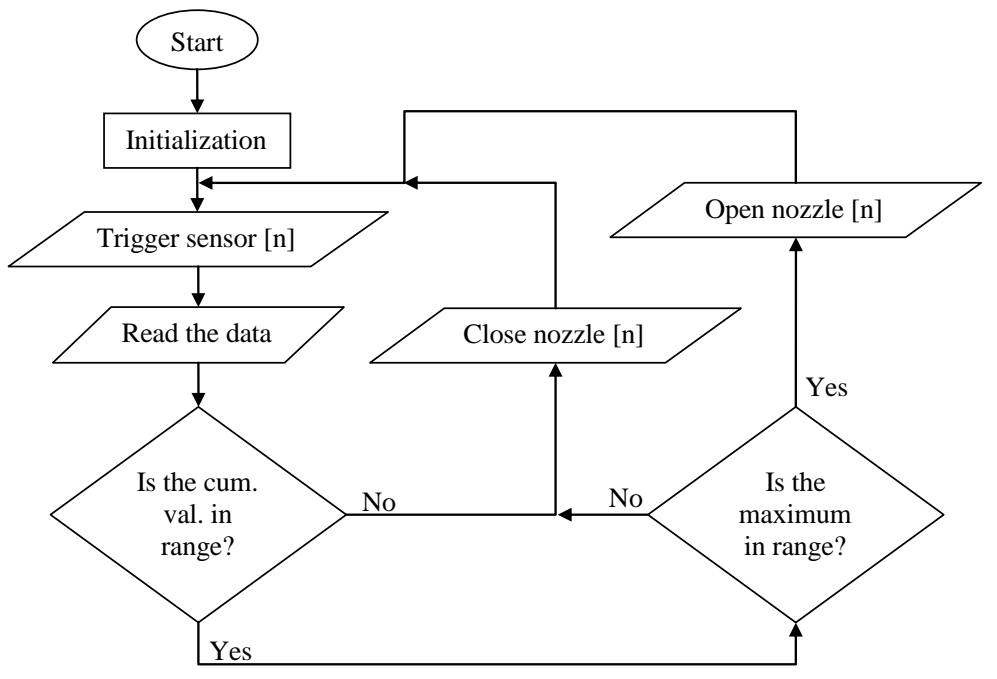

Fig 4. Simplified flow chart (without a delayed circular queue) of an algorithm running on the LPC1343 microcontroller

\subsection{Sensing System}

In order to control the operation of the sprayer autonomously the sprayer sensing system is tasked to determine the presence or the absence of a target (tree). The sensing system should include three or more ultrasonic sensors with programmable ultrasonic transceivers and the embedded computer described earlier. Each of the sensors sequentially generates a signal that is transmitted to a transceiver in order to produce an ultrasonic sound burst. The transceiver then waits to detect a reflected ultrasonic burst which contains information about canopy structure in a time series evolution of reflected intensity. When each sensor is commanded by one electrovalve the best accuracy for controlling the nozzle off/on is reported by many researchers. Simultaneously a bypass valve in the sprayer manifold usually allowed the sprayer to work also as a conventional sprayer. To avoid spraying at too low pressures, an antidrip device have to be mounted on each nozzle with an internal spring set to open at predicted working pressure i.e. 1.5 bar. These devices also help to shorten response times by keeping the pipes full, ready to spray when the pressure exceeded the one set with the springs.

\section{Conclusion}

The use of programmable microcontrollers enables the setting of a combination of threshold values for cumulative response and the integral of maximal response on different signals i.e. ultrasound. In many experiments the reductions from $79 \%$ to 59 $\%$, respectively, was reported in comparison with conventional spraying. These savings were achieved without significant reduction of tracer deposit at any tree position, in contrast to the significant reduction of spray deposit in areas between trees. Based on different field tests, significant reductions in operating costs ranged from $\$ 58$ per acre of peaches to $\$ 31$ per acre for prunes were reported. In younger prune orchards up to $40 \%$ can be saved. At the same time, the amount of pesticide in the running off water was reduced for 54\%. However, despite its obvious benefits in 
using sensor-based spray technology only a small part of the spraying equipment market remains to this newel sector. The main reason still lies in the sensor and control equipment costs, which reached about $15,000 €$ per sprayer. In contrast, assuming the huge pesticide savings estimated payback period of 2 years or less is expected in big plantations.

\section{References}

Cross, J.V., Walklate, P.J.; Murray, R.A. \& Richardson, G.M. (2001). Spray Deposits And Losses In Different Sized Apple Trees From An Axial Fan Orchard Sprayer: 1. Effects Of Spray Liquid Flow Rate. Crop Protection, 20, 13-30. ISSN: 0261-2194

Escolà, A.; Planas, S.; Rosell, J. R.; Pomar, J., Camp, F.; Solanelles, F.; Gracia, F.; Llorens, J. \& Gil, E. (2011). Performance of an Ultrasonic Ranging Sensor in Apple Tree Canopies. Sensors, 11, 2459-2477. ISSN 1424-8220

Gil, E.; Escolà, A.; Rosell, J.R.; Planas, S. \& Vald. L. (2007). Variable Rate Application Of Plant Protection Products In Vineyard Using Ultrasonic Sensors. Crop Protection, 26 (8), 1287-1297. ISSN: 0261-2194

Jejčič, V.; Godeša, T.; Hočevar, M.; Širok, B.; Malneršič, A.; Štancar, A.; Lešnik, M. \& Stajnko, D. (2011). Design and testing of an ultrasound system for targeted spraying in orchards. Journal of Mechanical Engineering, 57, 7-8, 587-598. ISSN: 0039-2480

Jeon, H.Y.; Zhu, H.; Derksen, R.; Ozkan, E. \& Krause, C. (2011). Evaluation of ultrasonic sensor for variable-rate spray applications. Computers \& Electronics in Agriculture, 75, 213-221. ISSN: 0168-1699

Koch, H. \& Weisser, P. (2000). Sensor equipped orchard spraying - efficacy, savings and drift reduction, Aspects of Applied Biology, 57, 357-362. ISSN: 0265-1491

Koch, H. (2007). How to achieve conformity with the dose expression and sprayer function in high crops, Pflanzenschutz-Nachrichten Bayer, 60 (1): 71-84. ISSN: 0340-1723

Llorens, J.; Gil, E.; Llop, J. \& Escolà, A. (2010). Variable rate dosing in precision viticulture: Use of electronic devices to improve application efficiency. Crop Protection, 29, 239-248. ISSN: 0261-2194

McConnell, R.L.; Elliot, K.C.; Blizzard, S.H. \& Koster, K.H. (1983). Electronic measurement of tree row volume. Computers \& Electronics in Agriculture, 1, 85-90. ISSN: 0168-1699

Moltó, E.; Martín, B. \& Gutiérrez, A. (2001). Pesticide Loss Reduction By Automatic Adoption Of Spraying On Globular Trees. Journal of Agricultural Engineering Research, 78, 35-41. ISSN: 0021-8634

Pai, N.; Salyani, M. \& Sweeb, R. D. (2009). Regulating airflow of orchard airblast sprayer based on tree foliage density, Transactions of the ASABE, 52 (5): 1423-1428. ISSN: 2151-0032

Palacin, J.; Pallejà, T.; Tresanchez, M.; Sanz, R.; Llorens, J.; Ribes-Dasi, M.; Masip, J.; Arnó, J.; Escolà, A. \& Rosell, J. R. (2007). Real-time tree-foliage surface estimation using a ground laser scanner, IEEE Transactions on Instrumentation and Measurement, 56 (4): 1377-1383. ISSN: 0018-9456 
Pergher, G. \& Petris, R. (2008). Pesticide Dose Adjustment In Vineyard Spraying And Potential For Dose Reduction. Agricultural Engineering International: the CIGR Ejournal. Manuscript ALNARP 08 011. Vol. X. May, 2008. ISSN: 1682-1130

Rosell, J. R. \& Sanz, R. (2012) A review of methods and applications of the geometric characterization of tree crops in agricultural activities, Computers and Electronics in Agriculture, 81: 124-141. ISSN: 0168-1699

Rovira-Más, F.; Zhang, Q.; Reid, J. F. \& Will, J. D. (2005). Hough-transform-based vision algorithm for crop row detection of an automated agricultural vehicle. Journal of Automobile Engineering, 219 (8), 999-1010. ISSN: 0954-4070

Sanz-Cortiella, R.; Llorens-Calveras, J.; Escolà, A.; Arnó-Satorra, J.; Ribes-Dasi, M.; Masip-Vilalta, J.; Camp, F.; Gràcia-Aguilá, F.; Solanelles-Batlle, F.; Planas-Demartí, S., Pallejà-Cabré, T.; Palacin-Roca, J.; Gregorio-Lopez, E.; Del-Moral-Martínez, I. \& Rosell-Polo, J. R. (2011). Innovative LIDAR 3D dynamic measurement system to estimate fruit-tree leaf area, Sensors, 11 (6), 5769-5791. ISSN: 1424-8220

Solanelles, F.; Escolà, A.; Planas, S.; Rosell, J. R.; Camp, F. \& Gràcia, F. (2006). An Electronic control system for pesticide application proportional to the canopy width of tree crops. Biosystems Engineering, 95, 473-481. ISSN: 1537-5110

Sutton, T.B. \& Unrath, C.R. (1988). Evaluation Of The Tree-Row-Volume Model For Full-Season Pesticide Application On Apples. Plant Disease, 72, 629-632. ISSN: 0191-2917

Tumbo, S.D.; Salyani, M.; Whitney, J.D.; Wheaton, T.A. \& Miller, W.M. (2002) Investigation of laser and ultrasonic ranging sensors for measurements of citrus canopy volume. Applied Engineering in Agriculture, 18, 3, 367-372. ISSN: 08838542

Walklate, P. J.; Cross, J. V.; Richardson, G. M. \& Baker, D. E. (2006) Optimising the adjustment of label-recommended dose rate for orchard spraying, Crop Protection, 25, 10, 1080-1086. ISSN: 0261-2194

Walklate, P. J.; Cross, J. V.; Richardson, G. M.; Murray, R. A. \& Baker, D. E. (2002). Comparison Of Different Spray Volume Deposition Models Using Lidar Measurements Of Apple Orchards. Biosystems Engineering, 82, 253-267. ISSN: $1537-5110$

Zande, J. C.; v. d., Achten, V. T. J. M.; Schepers, H. T. A. M.; Lans, A.; Kempenaar, C.; Michielsen, J.-M. G. P.; Stallinga, H.; Velde, P.; Oerke, E.-C.; Gerhards, R.; Menz, G. \& Sikora, R. A. (2010). Precision Disease Control in Bed-Grown Crops, In: Precision Crop Protection the Challenge and Use of Heterogeneity, Oerke, E.-C.; Gerhards, R.; Menz, G.; Sikora, R.A. (Eds.), 403-415, Springer Dordrecht Heidelberg London New York. ISBN 978-90-481-9277-2 